\title{
La interacción en el ámbito hospitalario según el cuidador familiar de pacientes con
} enfermedad crónica*

\author{
Interaction within the Hospital Environment According to Family Caretakers of Patients with Chronic Disease \\ A interação no âmbito hospitalário segundo o cuidador familiar de pacientes com doença crónica
}

Yadira Cardozo García ${ }^{a}$

DOI: https://doi.org/10.11144/Javeriana.ie20-1.iahc

Universidad de Antioquia, Colombia

yadira.cardozo@udea.edu.co

Carmen Liliana Escobar Ciro

Universidad de Antioquia, Colombia

Fecha de recepción: 27 Enero 2016 Fecha de aprobación: 10 Febrero 2017

\section{Resumen:}

Objetivo: describir las interacciones percibidas por el cuidador familiar en el ámbito hospitalario entre los miembros del equipo de este y el binomio paciente/cuidador. Metodología: cualitativa con enfoque etnográfico. Se realizaron catorce entrevistas a cuidadores familiares en dos instituciones de la ciudad de Medellín (Colombia). El análisis se hizo a partir de la lectura de los relatos, las observaciones y las notas de campo. Resultados: los cuidadores familiares identifican en la interacción que se establece en el ámbito hospitalario dos tipos: una que se da dentro del mismo equipo de salud, la cual se caracteriza por ser una relación vertical y jerárquica, y la que se da entre el equipo de enfermería con el binomio paciente/cuidador familiar, la cual tiene unos factores que pueden facilitar u obstaculizar el acercamiento. Conclusiones: las interacciones percibidas por el cuidador familiar que se dan en el ámbito hospitalario están mediadas por asuntos de tipo jerárquico y por la comunicación que se establece para lograr el cuidado.

Palabras clave: investigación cualitativa, cuidadores, enfermería, relaciones interpersonales.

\section{Abstract:}

Objective: To describe interactions perceived by family caretakers within the health team and between the patient and caregiver. Methodology: Qualitative research with ethnographic approach; fourteen interviews conducted with family caregivers in two institutions in the city of Medellin (Colombia). The analysis was performed from the narrations, observations, and field notes. Results: Family caregivers identify in the interaction that is established in the hospital setting two types: One that is given within the same health team, which is characterized by being a vertical and hierarchical relationship, and that which occurs between the team of nursing with the binomial patient / family caregiver, which has some factors that can facilitate or hinder the approach. Conclusions: Interactions perceived by the CF occur in the hospital environment, are mediated by hierarchical issues and by the communication that is established to achieve care.

Keywords: qualitative research, caretakers, nursing, interpersonal relations.

\section{Resumo:}

Objetivo: descrever as interações percebidas pelo cuidador familiar da equipe de saúde e entre ele e o binômio paciente/cuidador. Metodologia: qualitativa com enfoque etnográfico, se realizaram catorze entrevistas com os cuidadores familiares em duas instituições da cidade de Medellín (Colômbia). A análise se fez a partir da leitura dos relatos, as observações e as notas de campo. Resultados: os cuidadores familiares identificam na interação que se estabelece no âmbito hospitalário dois tipos: uma que se dá dentro da equipe de saúde, a qual se caracteriza por ser uma relação vertical e hierárquica, e, a outra, a que se dá entre a equipo de enfermagem com o binômio paciente/cuidador familiar, a qual tem uns fatores que facilitam e outros que obstaculizam a aproximação. Conclusões: as interações, percebidas pelo CF, estão mediadas por temas de tipo hierárquico e pela comunicação que se estabelece para conseguir o cuidado.

Palavras-chave: investigação qualitativa, cuidadores, enfermagem, relações interpessoais.

Notas de autor 


\section{Introducción}

Las enfermedades crónicas no transmisibles son afecciones de larga duración y de progresión lenta. Según la Organización Mundial de la Salud, en el Plan de Acción Mundial para la Prevención y el Control de las Enfermedades no Transmisibles 2013-2020, estas enfermedades causan el 63\% del total de muertes anuales en el mundo y cerca del 80\% se concentran en países de ingresos bajos y medios [1]. En Colombia, en el 2014 se calculó que el $71 \%$ del total de las muertes las causan enfermedades no transmisibles [2]. Estas aquejan, sobre todo, a personas de edad madura y ancianas y coexisten con otros padecimientos, razón por la cual la comorbilidad aumenta las tasas de hospitalización [1] e implica la presencia permanente de un cuidador familiar (CF) durante la estancia hospitalaria. En el presente artículo se retoma el concepto de Maeyama y colaboradores, quienes denominan $C F$ a aquella persona que presta atención a las personas que necesitan supervisión o asistencia en la enfermedad o la discapacidad. Ellos pueden proporcionar el cuidado en el hogar, en un hospital o en una institución; el concepto también hace referencia a padres, cónyuges u otros familiares y amigos [3].

Las personas que sufren enfermedades crónicas presentan una fragilidad clínica asociada a síntomas continuos y reagudizaciones frecuentes de su cuadro [4], situación que los lleva a consultas recurrentes y estancias hospitalarias habituales, que aumentan la demanda de cuidado de enfermería. El CF, en muchos casos, es quien asume el cuidado del paciente dentro del hospital, debido al vínculo familiar que los une. Es importante resaltar que el cuidado implica la interacción entre el personal de enfermería y el $\mathrm{CF} /$ persona cuidada. Como lo menciona Peplau [5], las relaciones enfermera-paciente las influyen experiencias previas, el lenguaje, los valores y los sentimientos. Para ella, el conocer las preconcepciones y expectativas del paciente hacia las enfermeras y la enfermería facilita la relación enfermera-paciente.

Los estudios realizados con los CF se relacionan, sobre todo, con la carga del cuidado, donde se indaga por aspectos emocionales, sociales, físicos, espirituales y aquellos que impactan la calidad de vida del cuidador $[6,7,8,9,10,11,12]$; pero poco se conoce de la percepción que tienen los cuidadores acerca del equipo de salud, en especial el personal de enfermería en el contexto hospitalario.

Teniendo en cuenta lo que implica el cuidado para un CF en el contexto hospitalario, y dadas sus condiciones, es importante que el equipo de enfermería se preocupe por comprender cómo los están visibilizando, pues, como ya se anotó, existe una preocupación constante respecto a cómo la enfermera es vista por el paciente, mas no por el CF, situación que puede interferir en la relación equipo de enfermería-paciente.

El presente artículo surge de la investigación Significados que construye el cuidador familiar de personas con enfermedad crónica acerca del personal de enfermería durante la estancia hospitalaria, donde uno de los objetivos específicos fue describir las interacciones percibidas por el cuidador familiar dentro del equipo de salud y entre enfermería y el binomio paciente/cuidador en el ámbito hospitalario. Lo anterior cobra importancia si las instituciones participantes tienen un modelo de atención centrado en el paciente y la familia. Los resultados favorecerán la creación de estrategias que faciliten la interacción, no desde la actividad instrumental del cuidado, sino desde una perspectiva de sujeto que cuida en la formalidad y otro que cuida en la informalidad, en beneficio del paciente.

\section{Metodología}

Para cumplir con el objetivo propuesto se abordó la investigación cualitativa desde el enfoque etnográfico, el cual permitió interpretar los fenómenos captando los significados que los participantes atribuyen a los hechos desde su subjetividad [13]. En relación con la selección de las instituciones, se hizo solicitud a dos instituciones hospitalarias de tercer y cuarto niveles de la ciudad de Medellín, que dieron su aval.

Los participantes se eligieron teniendo en cuenta que fueran CF de pacientes con enfermedad crónica hospitalizados en salas de medicina interna, para lo cual se aplicó el muestreo teórico planteado por Taylor 
y Bodgan [14]. Participaron catorce CF. Las técnicas usadas fueron la observación, la entrevista no dirigida y las notas de campo.

Se realizaron catorce entrevistas a los catorce CF que decidieron participar de la investigación de forma voluntaria, después de haberles presentado el proyecto, partiendo de una pregunta orientadora: ¿cómo se establece la relación CF y equipo de enfermería dentro del hospital? El número de entrevistas se llevó a cabo hasta la saturación de las categorías, es decir, cuando la información fue repetitiva y similar [15]. Cada entrevista tuvo una duración promedio de dos horas, se almacenaron en medio magnético y se identificaron con códigos alfanuméricos.

Durante el proceso investigativo, cada una de las investigadoras llevó un cuaderno con notas de campo donde se registró lo observado, sucesos, diálogos, interacciones y espacio físico. También se anotaron los comentarios útiles para el análisis.

Los resultados surgen del proceso de análisis de datos. Se hizo una lectura general de los relatos, se extractaron códigos que luego se agruparon en categorías y subcategorías, de forma manual y paralelo a su obtención. Con las categorías que emergieron se elaboraron mapas conceptuales que permitieron establecer algunas conexiones lógicas para identificar el significado que construye el personal de enfermería acerca del CF y facilitar así la escritura del informe final.

Para conservar el rigor metodológico se mantuvieron los criterios de credibilidad, confiabilidad y transferibilidad. La credibilidad se consiguió al obtener resultados verídicos y reales, sustentados en las entrevistas grabadas y transcritas textualmente, a fin de mantener la fidelidad de las expresiones de los participantes; la confiabilidad se consiguió al mantener la imparcialidad de las investigadoras en la interpretación y el análisis de la información; por último, la transferibilidad, ya que se tuvo en cuenta la posibilidad de aplicación del estudio y de extender los resultados a otros grupos con características similares.

Durante el proceso investigativo se cuidaron los aspectos éticos y las disposiciones consignadas en la Resolución 8430 de 1993 [16] para investigaciones de bajo riesgo, como el respeto a los participantes y la autonomía de decidir continuar o no en el estudio. Para proteger la privacidad y confidencialidad de los participantes y las instituciones se asignaron códigos a cada uno durante el análisis y la elaboración del informe final, y se estableció que los datos se utilizarían con fines académicos; también se contó con el aval de los comités de Ética de Investigación de la Facultad de Enfermería de la Universidad de Antioquia (Acta CEIFE 2013-31) y de las instituciones donde se recolectó la información (según actas 21-2013 e IN37-2013). Se devolvió la información a los CF y al personal de enfermería, quienes se identificaron con los resultados presentados.

\section{Resultados}

El presente artículo da cuenta de una de las categorías: interacción, compuesta por las subcategorías interacción dentro del equipo de salud e interacción entre el equipo de enfermería y el binomio paciente/cuidador. En la figura 1 se hace explícita la relación de la categoría interacción con sus respectivas subcategorías. 


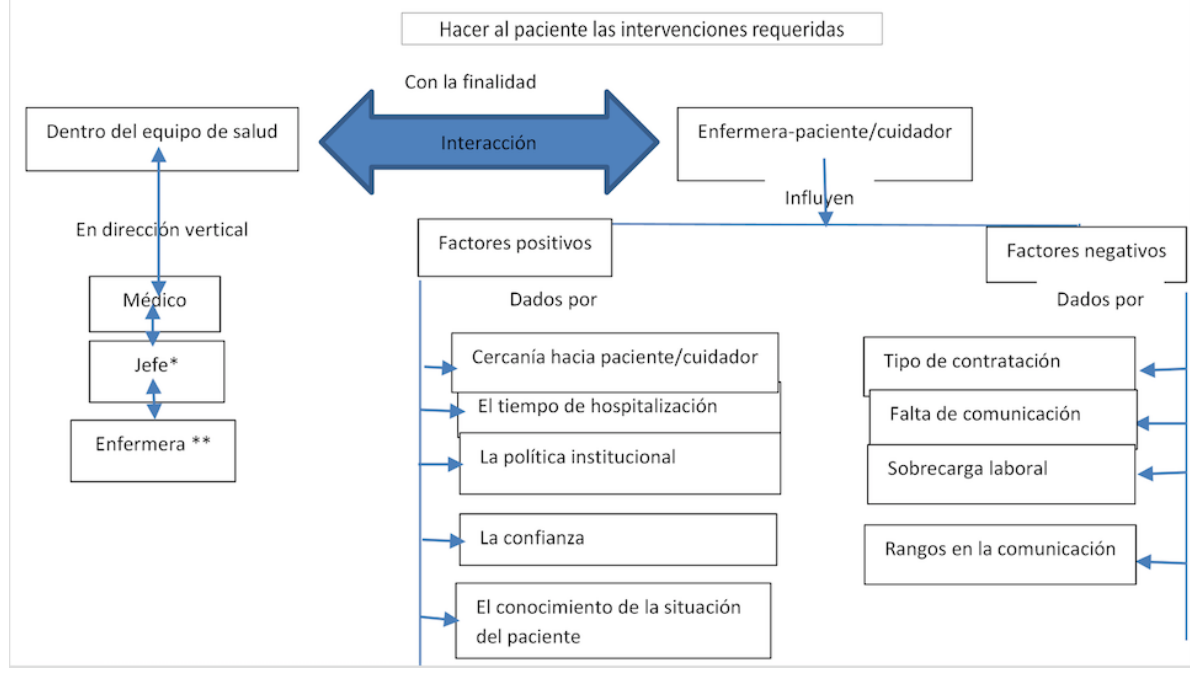

FIGURA 1

Relación de categorías y subcategorías

* Jefe: apelativo dado por los cuidadores al profesional de enfermería.

** Enfermera: apelativo dado por el cuidador familiar al auxiliar de enfermería.

Fuente: elaboración propia.

\section{Relación dentro del equipo de salud}

Dentro del equipo de salud, la relación se establece de forma unidireccional, esto es, se mantiene un orden jerárquico preestablecido según los relatos de los participantes, pues existen unos canales de comunicación que se deben seguir y que son dados a conocer desde el ingreso al hospital. Dicha jerarquía está relacionada con el nivel de formación y el cargo que se ocupa en la institución: “... con la primera persona que nos debemos comunicar es con la auxiliar que está a cargo de la paciente, ya luego con la jefe y después con el médico" (E1:1).

De igual forma, la enfermera, a quien los CF reconocen como jefe, es quien tiene a cargo a las auxiliares de enfermería (las enfermeras, según la percepción de los cuidadores); ella es quien las supervisa, les da órdenes, es considerada la principal del equipo de enfermería, además de ser la mano derecha del médico, quien debe recibir y cumplir las órdenes que este da: “... son las que son más directas al médico, son las que llaman al médico y le dicen está pasando esto y esto, y ellas ya reciben la información y reciben la instrucción que le dan a la auxiliar" (E5:2).

\section{Relación personal de enfermería con el binomio paciente-cuidador}

Por otro lado, está la relación que se establece entre el equipo de enfermería-paciente y el cuidador, en la cual influyen factores que permiten el acercamiento del cuidador, como la cercanía que se tenga hacia el paciente y cuidador, caracterizada por una relación familiar, por ser queridas, cariñosas, por el trato amable, por estar pendientes del paciente, por la entrega al ser humano, el ser especiales con el paciente y el cuidador; así se logra que la estancia hospitalaria sea más amena para estos:

Son muy buena gente y muy formales, buena gente es que te sonrían, que sean amables contigo, que si les preguntas algo de muy buenos modos te respondan, es lo que hacen acá, por ejemplo, yo he escuchado poquitas veces "ayyy, me esperas un momentico", pero al momentico están. (E8:4)

El tiempo de hospitalización es otro factor positivo que permite el acercamiento del cuidador al equipo de enfermería, pues sienten que cuando se lleva mucho tiempo en el hospital, se establece una relación más 
estrecha, y esto les permite tener mayores privilegios ("rosca") en relación con aquellos cuidadores que llevan poco tiempo en el hospital:

Pero como nosotros también las conocemos a casi todas y a todas las queremos y todas supuestamente nos quieren (risas) entonces ellas vienen pero es porque llevamos mucho tiempo acá, años, pero si fuera un paciente normal sería así un saludo y ya luego después lo que necesite, yo lo percibo; es más, si la jefe es nueva hace conmigo eso, me saluda por la mañana y ya. (E1:5)

Otro aspecto favorable es la política institucional, que en el caso de las instituciones participantes está centrada en el cuidado al paciente y su familia. De acuerdo con los relatos de los CF, el personal de enfermería tiene unas características que los hacen especiales al interactuar con el CF, como son la amabilidad, la prudencia o la capacidad de comunicarse, las cuales se consideran atributos humanos que debe tener el personal de enfermería para ser contratado por la institución: “... no sé cómo decir, pero sí son distintas, no sé si cuando las contratan les dicen "usted tiene que ser así con el paciente, usted tiene que ser muy amable”. No sé... Muy tratable, pienso yo... Tienen que hacerle alusión al logo" (E7:3).

La actitud hacia el cuidador es otro atributo importante para facilitar el acercamiento del CF al personal de enfermería y tiene una relación estrecha con la comunicación, pues el poder hablar con el personal y ser escuchados les da confianza y, a su vez, la confianza les permite acercarse durante el tiempo de hospitalización y establecer una relación más familiar y amigable, pues los hace sentir que su paciente está en buenas manos:

... me parece que son muy amigables y le dan a uno como esa confianza, que están como prestas a lo que uno necesite... Ellas, desde que llegan, se identifican: yo me llamo fulanita y soy la que voy a estar con usted, que dándole los medicamentos, y la otra: yo voy a estar para lo que necesiten, me timbran que nosotros venimos. (E7:1)

Otro aspecto facilitador es que el CF tenga los conocimientos acerca de la situación de salud del paciente. Los CF consideran que el estar ellos al cuidado de su ser querido y conocer cada detalle de la evolución médica les permite interactuar fácilmente con el personal de enfermería, ya que son ellos quienes pueden resolver las dudas e inquietudes que surjan durante el cuidado. El poseer información hace que sean tenidos en cuenta, que sean visibles para el personal de enfermería:

Me facilita porque puedo acercarme y ver en qué condiciones se encuentra mi mamá; en este caso me ayuda que tengo claro qué medicamentos y qué dosis le están colocando, qué cuidados debo tener con ella, cómo debo actuar antes las crisis que está sufriendo. (E13:2)

Dentro de los factores negativos para la interacción está el tipo de contratación del personal de enfermería, pues el estar vinculadas directamente a la institución y el tener estabilidad laboral hace que su actitud con el CF sea distinta, la perciben como una persona más querida, formal, habladora; en cambio, el estar contratados por cooperativas y no tener estabilidad laboral hace en algunos casos que sean malgeniadas, apáticas, groseras con el CF:

Que te respondan mal, uno les dice: "niña” y ahí mismo te gritan y te dicen que tienen mucho trabajo, que tienen más pacientes a su cargo, entonces que no les diga nada y más cuando me regañan, porque no baño a mi mamá o cuando se les acaba el suero, yo ni les aviso, y cuando vienen mero regaño: "oiga, que por qué no les dije y no les avisé que eso se terminó". Hasta una me salió con una cosa: "haaa es que nosotras trabajamos por cooperativa”. (E12:6)

Otra barrera que se presenta es la falta de comunicación, la cual se relaciona con la confianza que tiene el CF hacia el personal de enfermería. La confianza surge en el acto comunicativo y allí confluyen el lenguaje verbal y no verbal que utiliza el personal de enfermería durante la realización de las actividades del cuidado. Cuando el CF no encuentra un interlocutor, le es difícil interactuar y acercarse: “... vea, hay unas niñas que son muy formales y hay otras niñas como que son muy... Ni lo miran a uno, mejor decirle la verdad, entonces con qué confianza se le va arrimar uno a esa persona. Pienso yo" (E4:4).

Del mismo modo, la sobrecarga laboral es percibida como un obstáculo para la interacción, debido a las múltiples funciones que deben realizar las enfermeras - la responsabilidad por los pacientes y el cumplimiento de tareas propias de su rol, como son el computador y la papelería-, lo cual hace que 
estén más alejadas del paciente y cuidador. Para las auxiliares, debido al número de pacientes asignados, las interacciones se dan en momentos específicos, como son, según los $\mathrm{CF}$, el baño del paciente, la administración de medicamentos y la toma de signos vitales:

La diferencia es que el contacto no es el mismo, por decirlo así, ella está en su lugar... Lo que yo miro, pues, el contacto es solo de entrar y aquí estoy a la orden soy la enfermera jefe, cualquier inquietud con mucho gusto, y las auxiliares están más con el paciente, que lo está voltiando que le está aplicando el medicamento, que si ya desayunó, que venga yo le miro la presión, la azúcar todas esas cosas, que tenés un examen para ahorita, que no podés comer nada y todas esas cositas. $(\mathrm{E} 9: 1,9)$

Otra limitante en la interacción cuidador familiar-equipo de enfermería son los rangos que se establecen específicamente en la comunicación, lo cual hace que los CF se acerquen más fácilmente al auxiliar de enfermería por considerarse en iguales condiciones, y que la relación con la enfermera sea más distante, pues la ven como alguien de mayor estatus por su nivel de formación y ocupada en sus asuntos, es decir, en el puesto de enfermería, con los papeles y el computador; la perciben como alguien a quien pueden recurrir en situaciones complejas: "la jefe ya es más escaso; algo extremo que el paciente sí requiera más atención, porque ellas van y dan su visita y ya y listo; es lo que uno observa” (E3:4). La interacción CF-personal de enfermería tiene como finalidad lograr el cuidado del paciente durante la estancia hospitalaria.

\section{Discusión}

Los resultados sugieren que los CF perciben la interacción en dos niveles: uno de ellos se da a partir de las relaciones que se establecen entre los integrantes del equipo de salud, específicamente entre médico, enfermera y auxiliar de enfermería en el servicio de hospitalización; estas dependen del rol que cada uno asume en el equipo y por el cual son reconocidos por los CF. El otro nivel está dado por la cultura de la organización, que cobra importancia en esta interacción, la cual se entiende como la forma habitual de pensar y de actuar compartida por todos sus miembros. Esta tiene que ser aprendida y adoptada por las personas que se incorporan a la institución. La cultura se aprende, se comparte y se transmite [17]. Esta influye en la forma como el personal se relaciona entre sí, con los pacientes y con los CF.

Según los participantes, en el equipo de salud existe una relación jerárquica en la interacción, que se da básicamente en la comunicación y está muy relacionada con el nivel de formación, lo cual hace que las relaciones que allí se establecen sean verticales: el médico, seguido de la enfermera y, por último, la auxiliar de enfermería.

Según Alonso y colaboradores [18], la organización hospitalaria es la confluencia creciente entre disciplinas; es un sistema compuesto por múltiples elementos que mantienen entre sí una gran variedad de relaciones, con interacciones lineales y no lineales, que además pueden ser óptimas o complejas [19].

Para Thomas, citado por Linares y Reverte [20], a través de la historia el médico ha asumido una posición privilegiada y jerárquica dentro del equipo de salud, en las instituciones hospitalarias, lo cual puede explicarse por la formación que este recibe, enfocada en que él sea el líder del equipo. Dicha situación sigue presente en las instituciones hospitalarias actuales y de acuerdo con los relatos de los participantes del estudio. Coe, en su libro Sociología de la medicina [21], relata que el personal médico no solo dirige su práctica profesional, sino que controla también muchas otras actividades dentro de la institución; hoy en día, su influencia se extiende a muchos aspectos del funcionamiento del hospital como la enseñanza, la investigación, el establecimiento de normas para otro tipo de personal, etc.

La información y el conocimiento dentro del hospital representan y reproducen las relaciones de poder. Quero [22] nos habla de que el poder experto y el poder de la información lo representan los médicos; mientras que el poder de intermediación y de vinculación de las normas lo ejercen las enfermeras y las auxiliares. La información puede considerarse un patrimonio del profesional médico. Este papel es reforzado por las enfermeras y auxiliares, que en la práctica obstaculizan y limitan la relación entre el cuidador y el 
médico, según la misma autora. Así, en el imaginario de las personas, el médico es presentado como alguien lejano e inaccesible, cuya disponibilidad para el CF es poca, y este espacio es custodiado y vigilado por las enfermeras y auxiliares.

Para el caso de las enfermeras, los participantes las visibilizan en la interacción dentro del equipo de salud en su función dependiente del médico, que se enfoca en cumplir órdenes prescritas, lo cual tiene que ver con lo descrito por otros autores. Miralles, Burcet y Garreta [23] señalan que las funciones del personal de enfermería pueden ser: dependientes, cuando atienden problemas que son responsabilidad directa del médico, y donde ellas son quienes realizan las acciones delegadas; interdependientes, cuando atienden situaciones que deben ser resueltas en colaboración con otros profesionales, e independientes, en todas aquellas acciones que son reconocidas legalmente como responsabilidad directa de enfermería y que no requieren la supervisión o dirección de otros profesionales [23]. Las funciones interdependientes e independientes son difícilmente percibidas por los CF, pues en el servicio de hospitalización la enfermera pasa mucho tiempo realizando funciones de tipo administrativo y poco tiempo al lado del paciente y el CF.

El cuidado es un conjunto de interacciones, y cobra relevancia el ver cómo los CF no advierten la interacción con el profesional de enfermería lo que perciben como ausencia en el cuidado.

De igual forma, la profesional de enfermería mantiene una relación vertical con el personal auxiliar a su cargo, pues es ella la responsable del servicio, la encargada de hacer cumplir las órdenes dadas por el médico, de delegar el cuidado y supervisar el trabajo de estas, además de solucionar las dificultades que se presenten en la relación entre auxiliares de enfermería con los pacientes y los CF, a fin de realizar las intervenciones pertinentes. Quintero y Gómez [24] describen situaciones similares, donde el profesional de enfermería es considerado el principal responsable del cuidado de todos los pacientes, de que todo el servicio funcione bien; es quien designa las tareas al personal auxiliar, las supervisa y recibe las quejas de las faltas que cometen las auxiliares.

En un segundo nivel está la interacción que se da entre el equipo de enfermería y el binomio pacientecuidador, la cual depende de factores positivos y negativos que facilitan u obstaculizan la relación entre ambos actores; dentro de los factores positivos están la cercanía que se tenga hacia el paciente y el cuidador, las políticas institucionales, el tiempo de permanencia del CF en la institución, la actitud del personal hacia el cuidador y los conocimientos acerca del estado de salud del paciente que tenga el cuidador familiar.

Algo similar plantean Blanca y colaboradores [25]. Según los autores, las relaciones paciente-familia con los profesionales pueden ser distantes, cordiales o familiares, y estas dependen del tiempo de hospitalización, cada una con características específicas en la comunicación y la forma de relacionarse; se menciona, además, que a mayor tiempo de hospitalización, más cercanía entre el personal de salud y el paciente/cuidador. Esta interacción se caracteriza por los vínculos de amistad y la confianza que se genera entre ambos.

Ballesteros [26] plantea que la identificación del personal de enfermería permite al familiar del paciente hospitalizado diferenciar con quién puede establecer la comunicación; es importante resaltar que ellos expresan sentirse mejor al entablar la comunicación con el personal técnico de enfermería, por sentirlo más cercano; al contrario, con el profesional de enfermería la comunicación es distante y concreta, pero es la que le brinda datos más confiables, seguros y válidos, por el conocimiento que tienen derivado de su formación universitaria.

En su estudio, Delicado y colaboradores [27] encontraron que las actitudes de las enfermeras hacia los cuidadores familiares y su interacción se aprecian en las diversas actividades realizadas con ellos, en las relaciones que mantienen y en los factores que influyen y condicionan esta interacción.

Morales y colegas [28] expresaron que los familiares consideran la comunicación y la interacción como elementos fundamentales de la relación con el personal de enfermería y que no deben limitarse solo a la enseñanza del cuidado, sino también a la realización de intervenciones que los involucren y les den seguridad y ánimo. 
Además, en la relación personal de enfermería-CF se establecen unos vínculos durante la actividad cuidadora que están íntimamente relacionados con la cercanía y empatía que se dan durante el tiempo de hospitalización. En otras palabras, la interacción se construye a partir de la confianza que se establece entre personal de enfermería-paciente/cuidador [29].

Ahora bien, dentro de los factores que obstaculizan el acercamiento del cuidador familiar al personal de enfermería está la falta de comunicación, donde el lenguaje no verbal es tan importante como el verbal y constituyen un aspecto indispensable para lograr la interacción. Según García y colegas [30], los aspectos mejor valorados han sido los elementos de comunicación no verbal que utiliza el personal de salud durante el periodo de hospitalización de los pacientes (contacto ocular, actitud agradable) y la escucha activa.

Otra limitante es la jerarquía hospitalaria, específicamente en la comunicación, pues los CF deben seguir unos canales que se establecen desde su ingreso al servicio y que hacen que la relación con la enfermera y el médico sea lejana, diferente a lo que ocurre con la auxiliar de enfermería, con quien comparten actividades de cuidado y con quien interactúan durante el tiempo de hospitalización.

García y colaboradores [31] expresan que la presencia es destacada como otra característica común en gran parte de los estudios. Esta se entiende como la capacidad de las enfermeras de estar presentes, de "acompañar" a las personas enfermas que están a su cargo dentro de una función principal, teniendo presente su individualidad y necesidades. Para Guadalupe [32], el cuidador familiar se convierte en un apoyo fundamental para el cuidado del paciente en el hospital, ya que este continúa con el cuidado, tal como está acostumbrado a hacerlo en el hogar, teniendo en cuenta que la hospitalización se convierte en un desafío a su capacidad de adaptación, por estar inmerso en un medio extraño y con personas desconocidas, además de tener que adaptarse a las dinámicas propias de cada turno y al equipo de salud.

Otro aspecto que interfiere en la relación del CF con el equipo de enfermería es la sobrecarga laboral que tienen las enfermeras y auxiliares en el día a día y que es percibida por los CF como un factor negativo que no permite la interacción, pues ellas tienen muchos pacientes a cargo para el cuidado.

Ángel y colegas [33] expresan que la carga laboral afecta la disponibilidad de tiempo que se requiere para establecer contacto directo con los pacientes. Ello desvirtúa la esencia de la enfermería, que se fundamenta en una relación intersubjetiva y dialógica que favorece el crecimiento mutuo y la satisfacción de necesidades diversas. Existe poca relación comunicativa entre enfermera y paciente, ya que esta participa en el proceso salud-enfermedad; pero de una manera indirecta, dadas las múltiples funciones administrativas que tiene. Situación similar a la percibida por los CF.

En definitiva, la destreza en el manejo de la interacción se logra mediante el dominio de las técnicas de comunicación, de ahí la importancia de construir una relación adecuada desde el principio, sustentada en la confianza, la seguridad, el apoyo emocional, el tacto, la escucha, el confort, la ética, el respeto y la presencia cuidadora [34].

Mejía [35] nos recuerda que algunas de las características que debe tener la enfermera para lograr interactuar con el paciente y el cuidador familiar son: oír, comprender, dar respuestas, poseer sensibilidad intuitiva, brindar confianza, confidencialidad, permitir a los familiares expresar sentimientos, ideas y dudas; por ello se requieren sistemas de comunicación efectivos, considerando que la comunicación interpersonal es un proceso dinámico, que incluye el lenguaje verbal y no verbal en el momento de transmitir o recibir la información.

Es importante puntualizar que el cuidado brindado por el profesional de enfermería es un asunto cada vez más borroso, pues durante la estancia hospitalaria se responsabiliza cada vez al CF del cuidado básico de sus seres queridos, y a las auxiliares, de cuidados de mayor complejidad. Cabe recordar que el profesional de enfermería tiene la responsabilidad de brindar cuidado directo tanto físico como emocional, y en su relación con el CF debe integrarlos, apoyarlos y respetarlos en el cuidado que brindan a sus seres queridos [36]. 


\section{Conclusión}

Las interacciones percibidas por el CF en el ámbito hospitalario se dan en dos aspectos: la interacción que se establece dentro del equipo de salud, que se perciben mediadas en gran parte por la jerarquía hospitalaria y donde se establecen unas relaciones verticales incorporadas dentro de la cultura institucional, y la interacción entre el personal de enfermería con el paciente/cuidador, que está marcada por factores que se perciben como positivos, como confianza, tiempo, cercanía, conocimientos y políticas institucionales que facilitan el acercamiento, y aspectos considerados negativos y que sería necesario analizar más a fondo, como el tipo de contratación, la percepción de sobrecarga laboral, los rangos jerárquicos en la comunicación y ausencia de esta en algunos casos, que no permiten al CF acercarse para lograr la interacción.

Conflicto de interés

Ninguno de los autores manifiesta conflicto para la publicación de este artículo.

\section{Agradecimientos}

Artículo derivado del proyecto de investigación Significados que construye el cuidador familiar de personas con enfermedad crónica acerca del personal de enfermeria durante la estancia hospitalaria, financiado por el Comité para el Desarrollo de la Investigación (CODI), de la Universidad de Antioquia.

\section{Referencias}

1. Organización Mundial de la Salud. Plan de acción mundial para la prevención y el control de las enfermedades no trasmisibles 2013-2020 [internet]. Washington: OMS; 2013 [citado 2015 nov 18]. Disponible en: http://www .who.int/cardiovascular_diseases/15032013_updated_revised_draft_action_plan_spanish.pdf

2. Organización Mundial de la Salud. ENT: perfiles de países [internet]. Washington: OMS; 2014 [citado 2015 nov 18]. Disponible en: http://www.who.int/nmh/countries/col_es.pdf

3. Rivas JC, Ostiguin RM. Cuidador: ¿concepto operativo o preludio teórico? Enfer Univ [internet]. 2011 [citado 2015 nov 20];8(1):49-54. Disponible en: http://www.medigraphic.com/pdfs/enfuni/eu-2011/eu111g.pdf

4. Rico M, Sánchez S, Fuentelsaz C. El cuidado como elemento transversal en la atención a pacientes crónicos complejos Enferm Clin. 2014;24(1):44-50.

5. Peplau HE. Relaciones interpersonales en enfermería. Madrid: Salvat; 1990.

6. Sánchez B, Carrillo GM, Barrera L, Chaparro L. Carga del cuidado de la enfermedad crónica no transmisible. Aquichan. 2013;13(2):1-15.

7. Velásquez V, López L, Barreto Y. Cuidadores familiares campesinos: carga de cuidado, tiempo de cuidado y grado de funcionalidad. Investig Enferm Imagen Desarr. 2014;16(2):65-80. doi: 10.11144/Javeriana.IE16-2.cfcc

8. Carrillo GM, Chaparro L, Sánchez B. Carga del cuidado en cuidadores familiares de personas con enfermedad crónica en la región pacífica colombiana. Cienc Enferm. 2014;20(2):83-91.

9. Avilés MA, Morales M, Benavides MC, Reyna LL, Riquelme H, Ramírez JM, Barrón F, Gutiérrez R. Impacto de la parálisis cerebral en la carga y funcionalidad familiar. Rev Med Hosp Gen Méx. 2014;77(2):53-7.

10. Carrillo GM, Barreto RB, Arboleda LB, Gutiérrez OA, Melo BG, Ortiz VT. Competencia para cuidar en el hogar de personas con enfermedad crónica y sus cuidadores en Colombia. Rev Fac Med. 2015;63(4):665-75. doi: http:// dx.doi.org/10.15446/revfacmed.v63.n4.50322

11. Casado R, Ruiz E. Influence of gender and care strategy in family caregivers' strain: A cross-sectional study. J Nurs Scholarsh. 2016;48(6):587-97. doi: 10.1111/jnu 
12. Barrera L, Vargas E, Cendales PA. Encuesta de caracterización para el cuidado de una persona con enfermedad crónica. Investig Enferm Imagen Desarr. 2015;17(1): 27-43.

13. Joyceen B. Estilos de etnografía. En: Morse JM, editor. Asuntos críticos en los métodos de investigación cualitativa. Medellín: Universidad de Antioquia, Facultad de Enfermería; 2003.

14. Taylor SJ, Bogdan R. Introducción a los métodos cualitativos de investigación. Barcelona: Paidós; 1994.

15. Morse JM. The significance of saturation. Qual Health Res. 1995;5(2):147-9.

16. Resolución 008430 de 1993, por la cual se establecen las normas científicas, técnicas y administrativas para la administración en salud [Internet]. Bogotá: Ministerio de Salud [citado 2015 nov 5]. Disponible en: https://w ww.unisabana.edu.co/fileadmin/Documentos/Investigacion/comite_de_etica/Res_8430_1993_Salud.pdf

17. Marriner A. Guía de gestión y dirección de enfermería. Madrid: Elsevier; 2009.

18. Alonso E, Rubio A, March JC, Danet A. Clima emocional y comunicación interna en una unidad de gestión clínica y dos servicios hospitalarios tradicionales. Rev Calid Asist. 2011;26(5):281-4. doi: 10.1016/j.cali.2011.04.003

19. Moncin B. Análisis de la organización asistencial hospitalaria. En: Ferrer V, Medina JL, Lloret C. La complejidad en enfermería: profesión, gestión, formación. Barcelona: Laertes; 2003.

20. Linares M, Reverte M. Evaluación del trabajo en equipo en seis unidades de cuidados intensivos de dos hospitales universitarios. Enferm Intensiva. 2010;21(4):150-60.

21. Coe RM. Sociología de la medicina. Madrid: Alianza; 1979.

22. Quero A. Los cuidadores familiares en el hospital Ruiz de Alda de Granada [Tesis doctoral]. Granada: Universidad de Granada; 2007.

23. Miralles R, Burcet S, Garreta M. Equipo interdisciplinar en la atención geriátrica. En: Tratado de medicina geriátrica [internet]. Madrid: Elsevier; 2015 [citado 2015 nov 15]. Disponible en: https://www.clinicalkey.es/\#!/conten t/book/3-s2.0-B9788490221204000392

24. Quintero MT, Gómez M. El cuidado de enfermería significa ayuda. Aquichan [internet]. 2010 [citado 2015 nov 5];10(1):8-18. Disponible en: http://www.redalyc.org/articulo.oa?id=74116244002

25. Blanca JJ, Muñoz R, Caraballo MA, Expósito M, Sáez R, Fernández ME. La intimidad en el hospital: la experiencia de los pacientes, sus familias y las enfermeras. Index Enferm [internet]. 2008 [citado 2015 nov 20];17(2):106-10. Disponible en: http://scielo.isciii.es/scielo.php?script=sci_arttext\&pid=S1132-12962008000200006\&lng=es

26. Ballesteros G. Comunicación entre la enfermera y el familiar de la persona hospitalizada en la unidad de cuidados intensivos [Tesis Maestría en Enfermería]. Bogotá: Universidad Nacional de Colombia; 2014.

27. Delicado MV, Candel E, Alfaro A, López M, García C. Interacción de enfermería y cuidadoras informales de personas dependientes. Aten Primaria. 2004;33(4):195.

28. Morales MJ, Puerta AM, Gómez MM. La comunicación entre el personal de enfermería, el paciente y la familia en algunos servicios hospitalarios médico quirúrgicos. Actual Enferm. 2001;4(3):8-13.

29. Gaviria Noreña DL. La evaluación del cuidado de enfermería: un compromiso disciplinar. Invest Educ Enferm. 2009;27(1):24-33.

30. García JA, Abellón J, Celdrán AI, Manzanares JA, Satorres B. La importancia de la escucha activa en la intervención enfermera. Enferm Global. 2014;34:276-92.

31. García N, Errasti-Ibarrando B, Arantzamendi M. La relación enfermera-paciente con enfermedad avanzada y terminal: revisión bibliográfica y análisis conceptual. Med Paliat [internet]. 2014 [citado 2015 nov 20]. Disponible en: http://dx.doi.org/10.1016/j.medipa.2014.01.00

32. Guadalupe RM. Experiencia de los cuidadores de enfermos crónicos en el hospital. Enf Neurol. 2010;9(1):54-8.

33. Ángel G, López FE, Restrepo DM. La experiencia educativa del profesional de enfermería en el ámbito clínico. Invest Enferm Imagen Desarr. 2013;15(2):9-29.

34. Orkaizagirre A, Amezcua M, Huercanos I, Arroyo A. El estudio de casos, un instrumento de aprendizaje en la relación de cuidado. Index Enferm [internet]. 2014 [citado 2015 nov 20];23(4):244-9. Disponible en: http://w ww.index-f.com/index-enfermeria/v23n4/9903r.php 
Yadira Cardozo García, et al. La interacción en el ámbito hospitalario según el cuidador familiar ...

35. Mejía M. Interacción personal de enfermería-familiares paciente politraumatizado y su relación con el nivel de estrés de los familiares. Revista Electrónica de Portales Médicos [internet]. 2010 [citado 2015 nov 20] Disponible en: http://www.portalesmedicos.com/publicaciones/articles/2339/1/Interaccion-del-personal-de-Enfermeria -con-los-familiares-del-paciente-politraumatizado-y-su-relacion-con-el-nivel-de-estres-de-los-familiares.html

36. De la Cuesta Benjumea C. El cuidado del otro: desafíos y posibilidades. Invest Educ Enferm. 2007;25(1):106-12.

\section{Notas}

* Artículo de investigación.

\section{BY}

\section{Información adicional}

Cómo citar: Cardozo García Y, Escobar Ciro CL. La interacción en el ámbito hospitalario según el cuidador familiar de pacientes con enfermedad crónica. Investig Enferm Imagen Desarr. 2018;20(1):xx-xx. https://d oi.org/10.11144/Javeriana.ie20-1.iahc 